\title{
Condition or Disease under Study
}

National Cancer Institute

\section{Source}

National Cancer Institute. Condition or Disease under Study. NCI Thesaurus. Code C161319.

Primary disease(s) or condition(s) being studied in the trial, or the focus of the study.

(clinicaltrials.gov) 